\title{
antibiotics
}

ISSN 2079-6382

www.mdpi.com/journal/antibiotics

Article

\section{Identification of Additional Anti-Persister Activity against Borrelia burgdorferi from an FDA Drug Library}

\author{
Jie Feng, Megan Weitner, Wanliang Shi, Shuo Zhang, David Sullivan and Ying Zhang * \\ Department of Molecular Microbiology and Immunology, Bloomberg School of Public Health, \\ Johns Hopkins University, Baltimore, MD 21205, USA; E-Mails: jfeng16@jhu.edu (J.F.); \\ mweitne1@jhu.edu (M.W.); wshi3@jhu.edu (W.S.); shuozhang66@gmail.com (S.Z.); \\ dsulliv7@jhmi.edu (D.S.)
}

* Author to whom correspondence should be addressed; E-Mail: yzhang@jhsph.edu; Tel.: +1-410-614-2975.

Academic Editor: Leonard Amaral

Received: 2 July 2015 / Accepted: 10 September 2015 / Published: 16 September 2015

\begin{abstract}
Lyme disease is a leading vector-borne disease in the United States. Although the majority of Lyme patients can be cured with standard 2-4 week antibiotic treatment, $10 \%-20 \%$ of patients continue to suffer from prolonged post-treatment Lyme disease syndrome (PTLDS). While the cause for this is unclear, persisting organisms not killed by current Lyme antibiotics may be involved. In our previous study, we screened an FDA drug library and reported 27 top hits that showed high activity against Borrelia persisters. In this study, we present the results of an additional 113 active hits that have higher activity against the stationary phase B. burgdorferi than the currently used Lyme antibiotics. Many antimicrobial agents (antibiotics, antivirals, antifungals, anthelmintics or antiparasitics) used for treating other infections were found to have better activity than the current Lyme antibiotics. These include antibacterials such as rifamycins (3-formal-rifamycin, rifaximin, rifamycin SV), thiostrepton, quinolone drugs (sarafloxacin, clinafloxacin, tosufloxacin), and cell wall inhibitors carbenicillin, tazobactam, aztreonam; antifungal agents such as fluconazole, mepartricin, bifonazole, climbazole, oxiconazole, nystatin; antiviral agents zanamivir, nevirapine, tilorone; antimalarial agents artemisinin, methylene blue, and quidaldine blue; antihelmintic and antiparasitic agents toltrazuril, tartar emetic, potassium antimonyl tartrate trihydrate, oxantel, closantel, hycanthone, pyrimethamine, and tetramisole. Interestingly, drugs used for treating other non-infectious conditions including verteporfin, oltipraz, pyroglutamic acid, pidolic acid, and dextrorphan tartrate, that act on
\end{abstract}


the glutathione $/ \gamma$-glutamyl pathway involved in protection against free radical damage, and also the antidepressant drug indatraline, were found to have high activity against stationary phase B. burgdorferi. Among the active hits, agents that affect cell membranes, energy production, and reactive oxygen species production are more active against the $B$. burgdorfer $i$ persisters than the commonly used antibiotics that inhibit macromolecule biosynthesis. Future studies are needed to evaluate and optimize the promising active hits in drug combination studies in vitro and also in vivo in animal models. These studies may have implications for developing more effective treatments of Lyme disease.

Keywords: Borrelia burgdorferi; persisters; anti-persister activity; FDA drug library

\section{Introduction}

Borrelia burgdorferi is the causative agent of Lyme disease, the most common vector-borne disease in the United States and Europe. Although about 27,000 confirmed cases of Lyme disease in the United States were reported to the Centers for Disease Control and Prevention (CDC) in 2013, the total number of cases is estimated to be as high as 300,000 each year [1,2]. B. burgdorferi is transmitted during the blood feeding of Ixodes ticks on hosts including rodents, small mammals, and humans [3]. Lyme disease in humans is a multi-system disorder whose early stage is characterized by erythema migrans, a rapidly spreading rash that appears at the cutaneous site of infection in about $50 \%$ of patients [4]. Upon bacterial dissemination, patients can experience severe symptoms such as arthritis, carditis, and neurologic impairment [4].

The current treatment for Lyme disease is a 2-4 week antibiotic monotherapy with doxycycline, amoxicillin, or cefuroxime axetil [4]. However, according to the CDC, about $10 \%-20 \%$ of patients receiving this treatment experience chronic symptoms such as fatigue, muscle pain, and neurological impairment even six months after treatment [5], but a more recent study estimated the percentage of such patients to be at least 20\% [6]. Patients with these symptoms are diagnosed with Post-Treatment Lyme Disease Syndrome (PTLDS) and report significantly impaired functional ability and lower quality of life compared to Lyme patients without these symptoms [7]. The cause of PTLDS is unknown. Several theories have been proposed to explain this syndrome, including host response to continued presence of bacterial debris, autoimmunity, co-infections, and bacterial persisters not killed by the current Lyme antibiotics [8].

Evidence that supports the continued presence of persisting organisms despite antibiotic treatment has been well documented in various animal models such as mice, dogs, and nonhuman primates [9-12]. Intriguingly, the organism could not be cultured in conventional culture medium after antibiotic treatment but could be detected by more sensitive and indirect techniques such as xenodiagnosis and PCR. Similarly, in patients with chronic Lyme infections, signs of persisting organisms in a nonculturable form could be detected by positive PCR and xenodiagnosis [13]. Persistent bacteria are suggested as an explanation for the chronic symptoms of PTLDS as well as the observations of B. burgdorferi DNA without positive culturing results [14,15].

Persisters are a minor population of non-growing bacterial cells that are not killed by bactericidal antibiotic treatment $[16,17]$. Persisters are a heterogeneous bacterial population that are genetically drug 
susceptible but have phenotypic variations which allow them to survive in the presence of stressors such as antibiotics [17]. B. burgdorferi can change morphologies and develop persisters as the culture ages $[18,19]$. The log phase culture of B. burgdorferi consists primarily of spirochetes but round bodies and microcolonies become more abundant as the culture reaches stationary phase $[18,19]$. The current Lyme antibiotics, while having high activity against the spirochete log phase bacteria, show little activity against the stationary phase morphological variants which show features of persisters [18-20].

To identify drugs that target $B$. burgdorferi persisters, we recently screened a Food and Drug Administration (FDA) drug library and identified 165 hits with higher activity against B. burgdorferi persisters than the currently used Lyme antibiotics amoxicillin and doxycycline [18]. In that study, we reported the top 27 hits and some top hits were further evaluated in drug combination studies [18,19]. In this study, we present findings on the remainder of the 113 drug candidates from the FDA drug library with higher activity against the B. burgdorferi stationary phase culture than amoxicillin and doxycycline.

\section{Results and Discussion}

\subsection{Identification of Drug Candidates with High Anti-Persister Activity}

In our previous study, we identified 165 hits with higher activity against B. burgdorferi persisters than the currently used Lyme antibiotics. In this study, we further analyzed and characterized these active hits. By removing redundant hits from the library and also those that could not be repeated, we obtained 113 that gave consistent results (Supplementary Table S1). Of the 113 hits, the top 52 candidates that can be used in humans and killed $65 \%$ or more of the stationary phase bacteria according to either the SYBR Green I/Propidium Iodide (PI) assay or microscopic quantitation are presented in Table 1 . The remainder of active hits that may not be used in humans and also less active ones are presented in Supplementary Table S1. However, these agents in general were not as active as the top hits such as daptomycin, clofazimine, cefoperazone, and anthracyclines from the previous screens [18,21]. These active hits are grouped into antimicrobial agents (antibiotics, antivirals, antifungals, anthelmintics or antiparasitics), agents used for treating other disease conditions, as well as agents that may only be used as topical agents or not used internally and are presented below (Table 1).

We used a relatively high drug concentration of $50 \mu \mathrm{M}$ in our drug screens with seven-day-old stationary phase cultures; as in our preliminary pilot screens, lower drug concentrations, such as $10 \mu \mathrm{M}$, commonly used for actively growing organisms or protein target screens, would not allow one to see the effect of any compounds - even the highly active compounds such as daptomycin - under this condition. The findings with $50 \mu \mathrm{M}$ are still valid and relevant, because in the setting of drug combinations, the persister-active hits can be used at lower clinically relevant concentrations ( $10 \mu \mathrm{g} / \mathrm{mL}$ for daptomycin) and still allow one to see the effect and importance of the persister-active hits in eradicating more resistant microcolony forms of B. burgdorferi persisters [19]. In addition, taking into consideration some drugs such as pyrazinamide, with high activity against persisters in vivo but poor activity in vitro [17], we used this higher concentration to increase the sensitivity of the screen. We plan to validate these results using clinically relevant dosages in future drug combination studies in vitro and in vivo. 
Table 1. Top 52 active hits with better activity ( $p$-value $<0.05$ or live percentage by microscopy assay less than $68 \%$ ) against $B$. burgdorferi stationary phase cells than current Lyme antibiotics ${ }^{\mathrm{a}}$.

\begin{tabular}{|c|c|c|c|c|}
\hline Drugs $(50 \mu \mathrm{M})$ & Category & $\begin{array}{l}\text { Residual Viable Cells } \\
\text { (Microscopy) }^{\mathbf{b}}\end{array}$ & $\begin{array}{l}\text { Residual Viable Cells } \\
\text { (SYBR Green/PI) }^{\text {c }}\end{array}$ & $p$-Value ${ }^{\mathrm{d}}$ \\
\hline Control (no drug) & & $93 \%$ & $94 \%$ & - \\
\hline Doxycycline & Lyme antibiotic & $75 \%$ & $67 \%$ & 0.23360 \\
\hline Amoxicillin & Lyme antibiotic & $76 \%$ & $76 \%$ & 1.00000 \\
\hline Cefuroxime & Lyme antibiotic & $49 \%$ & $43 \%$ & 0.00032 \\
\hline Daptomycin & Antibiotic & $35 \%$ & $28 \%$ & 0.00001 \\
\hline Verteporfin $^{\mathrm{e}}$ & Ophthalmic & $47 \%$ & $27 \%$ & 0.00284 \\
\hline 3-formyl Rifamycin & Antibacterial & $59 \%$ & $42 \%$ & 0.00103 \\
\hline Tartar emetic & Anthelmintic & $45 \%$ & $42 \%$ & 0.00250 \\
\hline Toltrazuril & Antiprotozoal & $60 \%$ & $43 \%$ & 0.00296 \\
\hline Thiostrepton & Antibiotic & $66 \%$ & $43 \%$ & 0.00131 \\
\hline Mepartricin & Antifungal & $60 \%$ & $43 \%$ & 0.03214 \\
\hline Tilorone & Antiviral & & $44 \%$ & 0.04955 \\
\hline Oxantel & Anthelmintic & $63 \%$ & $44 \%$ & 0.01599 \\
\hline Pidolic acid $^{\mathrm{e}}$ & Antiseptic & $45 \%$ & $45 \%$ & 0.00477 \\
\hline Hycanthone & Anthelmintic & & $45 \%$ & 0.00154 \\
\hline Pyrimethamine & Antiprotozoal & $55 \%$ & $45 \%$ & 0.00030 \\
\hline Carbenicillin & Antibiotic & $64 \%$ & $46 \%$ & 0.06453 \\
\hline Oltipraz & Antitumor & $55 \%$ & $46 \%$ & 0.00121 \\
\hline Bitoscanate & Anthelmintic & & $46 \%$ & 0.00730 \\
\hline Sarafloxacin & Antibiotic & $50 \%$ & $47 \%$ & 0.05063 \\
\hline Bacitracin & Antibiotic & $60 \%$ & $47 \%$ & 0.06536 \\
\hline $\begin{array}{c}\text { Dextrorphan } \\
\text { tartrate }^{\mathrm{e}}\end{array}$ & Analgesic & $43 \%$ & $47 \%$ & 0.00361 \\
\hline Tetramisole & Anthelmintic & & $48 \%$ & 0.07051 \\
\hline Bifonazole & Antifungal & $50 \%$ & $48 \%$ & 0.09243 \\
\hline Ethacridine lactate & Antiseptic & & $48 \%$ & 0.04619 \\
\hline Zanamivir & Antiviral & $60 \%$ & $49 \%$ & 0.01224 \\
\hline Artemesinin & Antimalarial & $45 \%$ & $49 \%$ & 0.10432 \\
\hline Oxibendazole & Anthelminthic & & $51 \%$ & 0.00895 \\
\hline Indatraline $^{\mathrm{e}}$ & Antidepressant & $43 \%$ & $51 \%$ & 0.02578 \\
\hline Nevirapine & Antiviral & & $51 \%$ & 0.01604 \\
\hline Ganciclovir & Antiviral & & $53 \%$ & 0.04466 \\
\hline Phenothiazine & Anthelminthic & $53 \%$ & $54 \%$ & 0.00228 \\
\hline Oxfendazole & Anthelminthic & & $54 \%$ & 0.00095 \\
\hline Flubendazole & Anthelminthic & & $54 \%$ & 0.01183 \\
\hline Tazobactam & Antibiotic & $56 \%$ & $54 \%$ & 0.10052 \\
\hline Aztreonam & Antibiotic & $50 \%$ & $55 \%$ & 0.08105 \\
\hline Benzoylpas & Antibiotic & & $55 \%$ & 0.04017 \\
\hline
\end{tabular}


Table 1. Cont.

\begin{tabular}{|c|c|c|c|c|}
\hline Drugs (50 $\mu \mathrm{M})$ & Category & $\begin{array}{c}\text { Residual Viable Cells } \\
\text { (Microscopy) }^{\mathbf{b}}\end{array}$ & $\begin{array}{l}\text { Residual Viable Cells } \\
\text { (SYBR Green/PI) }^{c}\end{array}$ & $p$-Value ${ }^{\mathrm{d}}$ \\
\hline Fluconazole & Antifungal & $45 \%$ & $55 \%$ & 0.10643 \\
\hline Cefixime & Antibiotic & $56 \%$ & $56 \%$ & 0.03238 \\
\hline Sulfamoxole & Antibiotic & $55 \%$ & $57 \%$ & 0.02541 \\
\hline Tosufloxacin & Antibiotic & & $57 \%$ & 0.00067 \\
\hline Lamivudine & Antiviral & & $58 \%$ & 0.01121 \\
\hline Cefsulodin & Antibiotic & & $60 \%$ & 0.01463 \\
\hline Didanosine & Antiviral & & $61 \%$ & 0.02494 \\
\hline Floxuridine & Antiviral & & $61 \%$ & 0.01935 \\
\hline Cyacetacide & Antibacterial & & $61 \%$ & 0.03407 \\
\hline Oxiconazole nitrate & Antifungal & & $62 \%$ & 0.04957 \\
\hline Roxithromycin & Antibiotic & $65 \%$ & $62 \%$ & 0.15382 \\
\hline Ribavirin & Antiviral & & $63 \%$ & 0.00801 \\
\hline Griseofulvin & Antifungal & & $63 \%$ & 0.00957 \\
\hline Rifamycin sv & Antibiotic & $60 \%$ & $63 \%$ & 0.01872 \\
\hline Penciclovir & Antiviral & $60 \%$ & $64 \%$ & 0.09707 \\
\hline Nystatin & Antifungal & & $64 \%$ & 0.03312 \\
\hline Penimepicycline & Antibiotic & $60 \%$ & $65 \%$ & 0.00972 \\
\hline Puromycin & Antibiotic & $48 \%$ & $65 \%$ & 0.29297 \\
\hline Quinaldine blue & Antimalarial & $35 \%$ & Over range ${ }^{f}$ & - \\
\hline $\begin{array}{l}\text { Methylene blue } \\
\text { hydrate }\end{array}$ & Antimethemoglobinemic & $40 \%$ & Over range ${ }^{f}$ & - \\
\hline
\end{tabular}

${ }^{a}$ Stationary phase B. burgdorferi (seven day old) cells were treated with drugs for seven days. Daptomycin was used as a positive control with known high activity against $B$. burgdorferi persisters as shown previously. Drugs with live percentage of $B$. burgdorferi less than $65 \%$ by microscopy after drug exposure are presented in the table; ${ }^{b}$ Residual viable $B$. burgdorferi was assayed by epifluorescence microscope counting; ${ }^{\mathrm{c}}$ Residual viable $B$. burgdorferi was calculated according to the regression equation and ratio of green/red fluorescence obtained by SYBR Green I/PI assay. Three images of each sample were captured and quantitatively analyzed to determine the mean percent residual cells as indicated; ${ }^{\mathrm{d}} p$-values of the standard $\mathrm{t}$-test for the treated groups $(n=3) v s$. a control group treated with amoxicillin, which is known to have poor activity against stationary-phase persisters; ${ }^{\mathrm{e}}$ The italicized drugs are used to treat disease other than infection; ${ }^{\mathrm{f}}$ The value is over that of the drug-free control due to color of the compounds.

In our previous study, we compared the activity of antibiotics against non-growing persisters with their activity against growing $B$. burgdorferi [18]. Here we also tested the minimum inhibitory concentration (MIC) of some active hits. We found these drugs showed good activity against the growing B. burgdorferi with low MICs (Table 2). The maximum blood drug concentrations (Cmax) of several active hits, including 3-formyl rifamycin, oltipraz, and fluconazole, were higher than the MICs of these drug candidates, indicating these drugs are likely to achieve clinically relevant drug concentrations. However, some drugs such as verteporfin, pidolic acid, and dextrorphan tartrate had lower Cmax values than their corresponding MICs. Some other active hits such as thonzonium bromide, benzododecinium chloride, and quinaldine blue did not have Cmax values as they are used only topically or not available. 
Table 2. Minimum inhibitory concentration values of some persister-active hits for B. burgdorferi.

\begin{tabular}{ccc}
\hline Active Hits & MIC $(\boldsymbol{\mu g} / \mathbf{m L})$ & $\mathbf{C m a x}(\boldsymbol{\mu g} / \mathbf{m L})$ \\
\hline Verteporfin & $4.49-8.98$ & $1.03-1.14$ \\
Thonzonium Bromide & $0.92-1.85$ & NA \\
Benzododecinium Chloride & $0.60-1.20$ & NA \\
3-formyl Rifamycin & $2.27-4.54$ & 10 \\
Pidolic Acid & $0.81-1.61$ & 0.024 \\
Oltipraz & $0.71-1.41$ & 4.97 \\
Fluconazole & $0.48-0.96$ & $1.48-11.9$ \\
Dextrorphan Tartrate & $1.27-2.55$ & $0.025-0.030$ \\
Quinaldine Blue & $2.43-4.86$ & NA \\
\hline
\end{tabular}

NA: not available.

\subsection{Antimicrobial Agents with High Activity against Stationary Phase B. burgdorferi}

Readily available drugs with low toxicity are important objectives in this screen as they are the most likely to be used for the clinical treatment of Lyme disease. Here we focused on antimicrobial agents used in humans that had higher activity against the stationary phase $B$. burgdorferi than the commonly used Lyme antibiotics. The antibacterial agents include rifamycins (3-formal-rifamycin, rifaximin, rifamycin SV) (Figure 1), thiostrepton, quinolone drugs (sarafloxacin, clinafloxacin, tosufloxacin), carbenicillin, tazobactam, aztreonam, and puromycin (Table 1, Supplementary Table S1). Some antifungal agents such as fluconazole (Figure 1), mepartricin, bifonazole, climbazole, oxiconazole, and nystatin had reasonable activity against stationary phase B. burgdorferi (Table 1, Supplementary Table S1). Antiviral agents zanamivir, nevirapine, and tilorone (orally active interferon inducer) had good activity against stationary phase B. burgdorferi. Antimalarial agents artemisinin, methylene blue, and quidaldine blue were found to have good activity against stationary phase B. burgdorferi (Table 1). Antihelmintic and antiparasitic agents that had activity against $B$. burgdorferi included toltrazuril, tartar emetic, potassium antimonyl tartrate trihydrate, oxantel, closantel, hycanthone, pyrimethamine, and tetramisole (Table 1). These drugs with high activity against stationary phase $B$. burgdorferi in vitro are good potential candidates for drug combination studies and for further evaluation in animal models.

As previously published, the SYBR Green I/PI assay is a high-throughput technique that uses the ratio of green:red fluorescence in each sample to quantitate the amount of residual viable cells remaining [18]. While this technique has the benefits of high-throughput analysis, discoloration of the culture medium by test drugs can result in altered readings [18]. Quinaldine blue and methylene blue are two drugs whose staining properties resulted in medium discoloration and required verification through microscopy. Careful microscopy analysis revealed that quinaldine blue and methylene blue had high activity against $B$. burgdorferi persisters (Table 1, Figure 1). Quinaldine (2-methylquinoline) is a heterocyclic quinoline compound that is used as an antimalarial and in dye manufacturing, food colorants, $\mathrm{pH}$ indicators, and pharmaceuticals. Methylene blue was originally used as an antimalarial and is used to treat methemoglobinemia and urinary tract infections. 

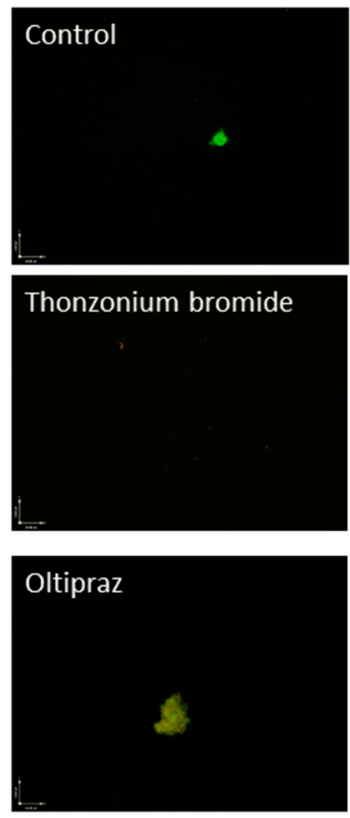
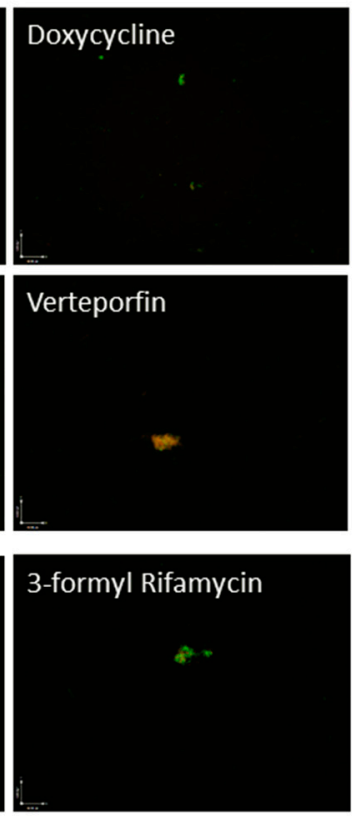
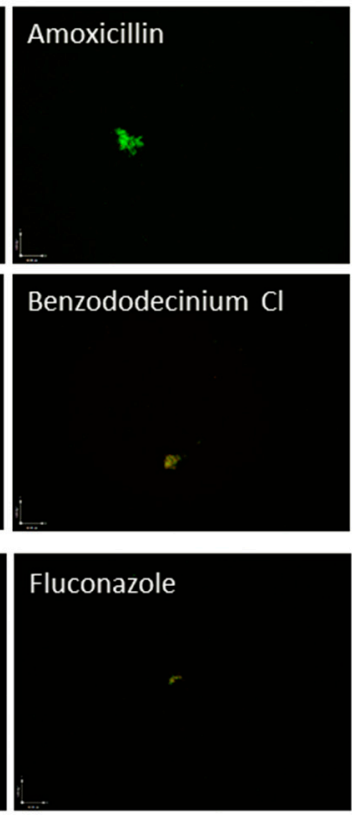

Daptomycin
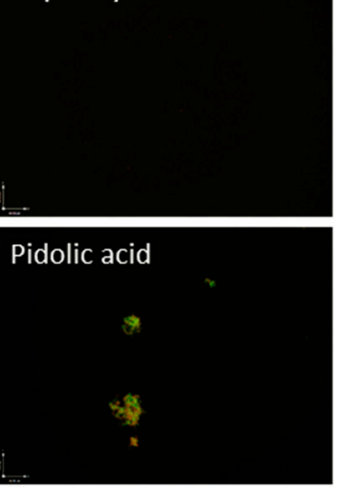

Quinaldine blue

Figure 1. Image of B. burgdorferi stationary phase culture (seven day old) incubated for seven days with the indicated drugs, stained by SYBR Green I/PI assay, and examined using epifluorescence microscopy. Live cells are indicated by green fluorescence and dead cells are indicated by red fluorescence.

Zanamivir is a clinically used antiviral agent that inhibits the neurominidase inhibitor that is inhaled as an aerosol to shorten the duration of influenza infections by preventing neuraminidase from releasing virions from the infected cells [22]. Recently, multiple bacterial species have been shown to express bacterial neuraminidases capable of cleaving $\alpha 2,3$-sialic acids [23]. These neuraminidases have been implicated in biofilm formation, with a $P$. aeruginosa neuraminidase mutant showing decreased ability to colonize the mouse respiratory tract and decreased biofilm production [23]. It remains to be seen if zanamivir acts in a similar manner in B. burgdorferi.

\subsection{Agents Used for Treating Other Disease Conditions}

It is interesting to note that several highly active drugs identified in our screen, including verteporfin, oltipraz, pyroglutamic acid, pidolic acid (Figure 1), and dextrorphan tartrate, act on the glutathione/ $\gamma$-glutamyl pathway used in mammalian cells which involved in protection against intracellular damage from free radicals and peroxides. Glutathione (GSH) is a reducing agent produced in the cytoplasm and transferred to the mitochondria by glutathione-S-transferase (GST), where it protects the mitochondria from reactive oxygen species (ROS) damage and functions in amino acid transport $[24,25]$. Reduced levels of GSH have been linked to increased sensitivity to ROS damage, resulting in mitochondrial swelling and subsequent damage [24,26].

Verteporfin (Visudyne), a benzophorphyrin derivative, is a photosensitizing agent currently used to treat the macular degeneration that affects the $\gamma$-glutamyl pathway [24,27,28]. This intravenous drug is transported in oxygenated blood by lipoproteins, and is activated by laser light treatment allowing for precise chemotherapeutic application [24]. Verteporfin is a possible effector of cell membrane permeability through ROS lipid peroxidation [24,29]. Activated verteporfin has been shown to target the 
mitochondria, producing reactive oxygen radicals and nitric oxide that damage local endothelium and seal leaky vessels [24,27]. Verteporfin depletes GSH levels in HepG2 cells after activation, possibly through increased nitric oxide production [24]. Oltipraz is an organosulfur compound that belongs to the dithiolethione class. It has been shown to inhibit schistosome and prevent the formation of cancer. It activates phase II detoxification enzymes in mammalian cells, which results in the binding of glutathione to electrophilic compounds and subsequent protection against reactive oxygen species (ROS) damage [30,31]. Pyroglutamic acid (PCA) or pidolic acid (pidolate) or 5-oxoproline is an amino acid derivative that is involved in the $\gamma$-glutamyl cycle. PCA is a metabolite of the glutathione cycle which is broken down to glutamate and cysteine, which are then converted back into glutathione [26], and is used in humans as dietary supplement and skin moisturizer retainer.

The significant number of highly active drugs that act upon this $\gamma$-glutamyl pathway suggests that this pathway is important for persisters and that ROS and peroxide-induced damage are important for killing persisters. We anticipate that inhibition of this pathway could be a good therapeutic target for B. burgdorferi persisters for the improved treatment of Lyme disease. Future studies are needed to further evaluate if these agents are active in drug combination studies and in animal models.

\subsection{Active Hits that Are Topical Agents or Toxic for Internal Use}

Thonzonium bromide, benzododecinium chloride, and butyl chloride were found to have very high activities against stationary phase B. burgdorferi (Supplementary Table S1, Figure 1). Thonzonium bromide even had comparable activity to daptomycin against stationary phase B. burgdorferi. However, thonzonium bromide is a cationic detergent and surfactant that is used as a topical agent in combination with other compounds to assist in the penetration of cellular membranes [32]. Thonzonium bromide has been shown to inhibit vacuolar ATPases in yeast, which is an enzyme that is closely related to the ATPase found in B. burgdorferi [33-35]. In C. albicans, thonzonium bromide was also shown to inhibit ATPases in isolated vacuoles and cause general cellular toxicity [33,34]. Thonzonium bromide was also shown to be active against preformed $C$. albicans biofilms [32]. Benzododecinium chloride is a C12-substituted alkyl chain derivate of the quarternary ammonium detergent benzalkonium chloride that alters cell membrane permeability and can cause cell lysis through lipid dispersion [36,37]. Benzododecinium chloride was shown in $S$. aureus to have higher activity against the biofilm form of the bacteria than the free planktonic form in vitro [38]. Since thonzonium bromide and benzododecinium chloride have strong detergent properties causing generalized cellular damage in humans, they may not be used directly for Lyme treatment. However, the high activity of these drugs against $B$. burgdorferi persisters suggests that both the cell membrane and biofilms are potential targets for future persister drug design.

It is worth noting that the most active hits from the compound library screen are those that affect cell membranes (benzododecinium chloride, thonzonium bromide, zanamivir). This is consistent with our previous finding that daptomycin and clofazimine may act on the cell membrane to show their high activity against $B$. burgdorferi persisters [18]. Indeed, agents that target bacterial cell membranes have been found to be active against persisters in different bacterial pathogens such as $M$. tuberculosis and E. coli [39-41]. Other active hits that show good activity against B. burgdorferi persisters interfere with energy production (thonzonium bromide, oxantel) and ROS production (verteporfin, oltipraz, 
pyroglutamic acid, pidolic acid). Our data showed that these three types of agents, cell membrane disruptors, energy inhibitors, and ROS producers, are generally more active against the $B$. burgdorferi persisters than the more conventional antibiotics that inhibit cell wall, protein, RNA, and DNA syntheses (Table 1, Supplementary Table S1). Future studies are needed to assess the activity of these agents in combination with Lyme antibiotics for more effective eradication of B. burgdorferi persisters in vitro [19].

Efflux pumps of $B$ burgdorferi persisters have not received any attention other than in the structural study of the adaptor protein of the tripartite efflux pump of the organism. Given our results in this study and also our recent observation that efflux and transporters are upregulated in B. burgdorferi persisters [42], it is very likely that over-expressed efflux pumps are one of the causes for the persisters and that compounds known to inhibit bacterial efflux pumps such as RND proton motive force-dependent pumps are possible lead compounds for the development of effective drugs for the treatment of persisting Lyme disease.

A recent study by Sharma et al. used colony-forming unit (CFU) assay to assess "persisters" in B. burgdorferi [43]. However, the CFU assay, while working well for bacteria that easily form colonies on agar plates, does not work well for B. burgdorferi and is not commonly used for viability assessment for $B$. burgdorferi. Instead, BacLight LIVE/DEAD viability assay is the most commonly used method for assessing drug activity in this field. In addition, it is well known that true B. burgdorferi persisters after antibiotic treatment in vivo cannot be cultured in Barbour-Stoenner-Kelly (BSK) media, and therefore the CFU assay may not be an appropriate method to evaluate the survival of persisters from in vivo. Furthermore, the CFU assay may be used only on relatively young cultures (3-5 days) that consist primarily of planktonic spirochetal forms, but cannot be used for older true stationary phase cultures (7-10 days) that consist mainly of aggregated microcolonies [19]. Therefore, we did not use CFU assay in our studies involving seven-day-old cultures, but instead used SYBYR Green I/PI viability assay, which is similar in principle but more sensitive than BacLight LIVE/DEAD viability assay [44]; we also used subculture in liquid medium to assess the anti-persister activity of the drugs. In contrast to the CFU assay, the SYBYR Green I/PI viability assay and subculture in liquid medium can be readily used and are more suited to this setting. In addition, the CFU assay cannot be used for drug screens as it is not only infeasible due to the washing to remove drugs for the CFU assay in a high-throughput format, but it also requires a significant amount of drugs, which precluded us from testing the active hits with the CFU assay, as the FDA drug library did not have a sufficient amount for it. Nevertheless, the CFU assay will be performed in future studies on selected active hits using younger cultures with primarily planktonic spirochetes when a sufficient quantity of the drugs is secured for testing.

Drug screens are subject to false positive non-specific hits referred to as pan-assay interference compounds (PAINS) in protein target-based screens [45]. However, because this study used whole organism (stationary phase culture)-based screens, most of the factors that can lead to PAINS are not present in our assay system. Nevertheless, we looked into the possible presence of PAINS in our active hits derived from the FDA drug library using the software from the Scripps Institute program for chemical promiscuity evaluation [46]. We found the majority of our hits to be highly specific, and the only identified PAINS in our highly active hits included FDA-approved drugs that are also coloring chemicals including methylene blue, quinaldine blue, and ethacridine lactate. The possibility of the PAINS was excluded using manual verification of the anti-persister activity of these drugs through epifluorescence microscopy. 
The current study has significant limitations as it looks at the effect of these compounds in a cell-free culture system. Whether these active hits have efficacy in vivo is influenced by many factors such as drug absorption, appropriate blood and tissue drug concentrations, tissue penetration and tropism, immune reactivity, and other factors that are missing from the cell-free system. Further studies are needed to confirm if the active hits are effective in vivo in animal models before human studies.

\section{Experimental Section}

\subsection{Strain and Culture Techniques}

Borrelia burgdorferi strain B31 (ATCC35210) was received from the American Type Tissue Collection (Manassas, VA, USA) and was grown in BSK-H medium (HiMedia Laboratories, Mumbai, India) and 6\% rabbit serum (Sigma Aldrich, St. Louis, MO, USA). The culture was filtered and sterilized using a $0.2 \mathrm{~mm}$ filter and incubated in capped sterile $50 \mathrm{~mL}$ conical tubes (BD Biosciences, San Jose, CA, USA) at $33{ }^{\circ} \mathrm{C}$ for seven days without antibiotics until the culture reached stationary phase. Seven-day-old stationary phase cultures were transferred to a 96-well culture plate for evaluation of drugs on active $B$. burgdorferi persisters.

\subsection{Microscopy}

The cultures were examined using a Nikon Eclipse E800 microscope with differential interference contrast and epifluorescent illumination. The pictures were captured using a SPOT slider color camera. A SYBR Green I/PI assay was used to assess the viability of the bacterial sample using the ratio of live to dead B. burgdorferi (measured with green and red fluorescence, respectively) as measured by a plate reader. The cellular counts were made by counting 100-200 cells per image based on three images representative of the bacterial samples using epifluorescence microscopy and quantitatively analyzed using Image Pro-Plus software to calculate the fluorescence intensity as described [44].

\subsection{Drug Library Screens for Activity against B. burgdorferi Persisters in Vitro}

The FDA drug library screens against the stationary phase B. burgdorferi persister model were performed as described [18]. Briefly, prediluted drug stock $(10 \mu \mathrm{L})$ was added to seven-day-old stationary phase $B$. burgdorferi culture $(90 \mu \mathrm{L})$ to achieve a $50 \mu \mathrm{M}$ final drug concentration per well. The plates were then incubated at $33^{\circ} \mathrm{C}$ for seven days, at which point the SYBR/PI rapid viability assay was performed in a fluorescence plate reader to obtain the green-red fluorescence ratio. The top hits from the SYBR Green I/PI assay were then examined using epifluorescence microscopy to ensure accuracy of the SYBR Green I/PI readings and to ensure no fluorescent contamination from colored test drugs as described previously [18].

\section{Conclusions}

In this study we identified 113 active hits that have higher activity against the stationary phase B. burgdorferi than the currently used Lyme antibiotics. These active hits include commonly used antimicrobials for treating other infections as well as some agents that are used for treating other disease 
conditions. Agents that affect cell membranes, energy production, and ROS production are generally more active against the B. burgdorferi persisters than the commonly used antibiotics that inhibit macromolecule biosyntheses. Future studies are needed to evaluate and optimize the promising active hits in drug combination studies in vitro and also in vivo in animal models. These studies may have implications for the improved treatment of Lyme disease.

\section{Acknowledgments}

This work was supported by the Global Lyme Alliance (formerly Lyme Research Alliance). Y.Z. was supported in part by Global Lyme Alliance and NIH grants AI099512 and AI108535.

\section{Author Contributions}

Ying Zhang conceived the experiments; Jie Feng, Megan Weitner, Wanliang Shi, Shuo Zhang, David Sullivan, performed the experiments; Jie Feng, Megan Weitner, David Sullivan, and Ying Zhang analyzed the data; and Megan Weitner, Jie Feng, Ying Zhang wrote the paper.

\section{Conflicts of Interest}

The authors declare no conflict of interest.

\section{References}

1. Centers for Disease Control and Prevention. Lyme Disease. Available online: http://www.cdc.gov/lyme/ (accessed on 5 June 2015).

2. Hinckley, A.F.; Connally, N.P.; Meek, J.I.; Johnson, B.J.; Kemperman, M.M.; Feldman, K.A.; White, J.L.; Mead, P.S. Lyme disease testing by large commercial laboratories in the United States. Clin. Infect. Dis. 2014, 59, 676-681.

3. Radolf, J.D.; Caimano, M.J.; Stevenson, B.; Hu, L.T. Of ticks, mice and men: Understanding the dual-host lifestyle of lyme disease spirochaetes. Nat. Rev. Microbiol. 2012, 10, 87-99.

4. Wormser, G.P.; Dattwyler, R.J.; Shapiro, E.D.; Halperin, J.J.; Steere, A.C.; Klempner, M.S.; Krause, P.J.; Bakken, J.S.; Strle, F.; Stanek, G.; et al. The clinical assessment, treatment, and prevention of lyme disease, human granulocytic anaplasmosis, and babesiosis: Clinical practice guidelines by the infectious diseases society of america. Clin. Infect. Dis. 2006, 43, 1089-1134.

5. Centers for Disease Control and Prevention. Post Treatment Lyme Disease. Available online: http://www.cdc.gov/lyme/postLDS (accessed on 5 June 2015).

6. Adrion, E.R.; Aucott, J.; Lemke, K.W.; Weiner, J.P. Health care costs, utilization and patterns of care following lyme disease. PLoS ONE 2015, 10, e0116767.

7. Aucott, J.N.; Rebman, A.W.; Crowder, L.A.; Kortte, K.B. Post-treatment lyme disease syndrome symptomatology and the impact on life functioning: Is there something there? Qual. Life Res. 2013, $22,75-84$.

8. Phillips, S.E.; Mattman, L.H.; Hulinska, D.; Moayad, H. A proposal for the reliable culture of Borrelia burgdorferi from patients with chronic lyme disease, even from those previously aggressively treated. Infection 1998, 26, 364-367. 
9. Barthold, S.W.; Hodzic, E.; Imai, D.M.; Feng, S.; Yang, X.; Luft, B.J. Ineffectiveness of tigecycline against persistent Borrelia burgdorferi. Antimicrob. Agents Chemother. 2010, 54, 643-651.

10. Embers, M.E.; Barthold, S.W.; Borda, J.T.; Bowers, L.; Doyle, L.; Hodzic, E.; Jacobs, M.B.; Hasenkampf, N.R.; Martin, D.S.; Narasimhan, S.; et al. Persistence of Borrelia burgdorferi in Rhesus macaques following antibiotic treatment of disseminated infection. PLoS ONE 2012, 7, e29914.

11. Hodzic, E.; Imai, D.; Feng, S.; Barthold, S.W. Resurgence of persisting non-cultivable Borrelia burgdorferi following antibiotic treatment in mice. PLoS ONE 2014, 9, e86907.

12. Straubinger, R.K.; Summers, B.A.; Chang, Y.F.; Appel, M.J. Persistence of Borrelia burgdorferi in experimentally infected dogs after antibiotic treatment. J. Clin. Microbiol. 1997, 35, 111-116.

13. Marques, A.; Telford, S.R., 3rd; Turk, S.P.; Chung, E.; Williams, C.; Dardick, K.; Krause, P.J.; Brandeburg, C.; Crowder, C.D.; Carolan, H.E.; et al. Xenodiagnosis to detect Borrelia burgdorferi infection: A first-in-human study. Clin. Infect. Dis. 2014, 58, 937-945.

14. Hodzic, E.; Feng, S.; Holden, K.; Freet, K.J.; Barthold, S.W. Persistence of Borrelia burgdorferi following antibiotic treatment in mice. Antimicrob. Agents Chemother. 2008, 52, 1728-1736.

15. Bayer, M.E.; Zhang, L.; Bayer, M.H. Borrelia burgdorferi DNA in the urine of treated patients with chronic lyme disease symptoms. A PCR study of 97 cases. Infection 1996, 24, 347-353.

16. Lewis, K. Persister cells. Annu. Rev. Microbiol. 2010, 64, 357-372.

17. Zhang, Y. Persisters, persistent infections and the yin-yang model. Emerg. Microbes Infect. 2014, 3, doi:10.1038/emi.2014.3.

18. Feng, J.; Wang, T.; Shi, W.; Zhang, S.; Sullivan, D.; Auwaerter, P.G.; Zhang, Y. Identification of novel activity against Borrelia burgdorferi persisters using an FDA approved drug library. Emerg. Microbes Infect. 2014, 3, doi:10.1038/emi.2014.1053.

19. Feng, J.; Auwaerter, P.G.; Zhang, Y. Drug combinations against Borrelia burgdorferi persisters in vitro: Eradication achieved by using daptomycin, cefoperazone and doxycycline. PLoS ONE 2015, 10, e0117207.

20. Sapi, E.; Kaur, N.; Anyanwu, S.; Luecke, D.F.; Datar, A.; Patel, S.; Rossi, M.; Stricker, R.B. Evaluation of in vitro antibiotic susceptibility of different morphological forms of Borrelia burgdorferi. Infect. Drug Resist. 2011, 4, 97-113.

21. Feng, J.; Shi, W.; Zhang, S.; Zhang, Y. Identification of new compounds with high activity against Borrelia burgdorferi persisters from the nci compound collection. Emerg. Microbes Infect. 2015, 4, doi:10.1038/emi.2015.31.

22. US National Library of Medicine. Zanamivir Oral Inhalation. Available online: http://www.nlm.nih.gov/medlineplus/druginfo/meds/a699021.html (accessed on 24 June 2015).

23. Soon, G.; Muir, A.; Gomez, M.I.; Waks, J.; Reddy, B.; Planet, P.; Singh, P.K.; Kanetko, Y.; Wolfgang, M.C.; Hsiao, Y., et al. Bacterial neuraminidase facilitates mucosal infection by participating in biofilm production. J. Clin. Investig. 2006, 116, 2297-2305.

24. Chiou, J.F.; Wang, Y.H.; Jou, M.J.; Liu, T.Z.; Shiau, C.Y. Verteporfin-photoinduced apoptosis in HepG2 cells mediated by reactive oxygen and nitrogen species intermediates. Free Radic. Res. 2010, 44, 155-170.

25. Tate, S.S.; Ross, L.L.; Meister, A. The gamma-glutamyl cycle in the choroid plexus: Its possible function in amino acid transport. Proc. Natl. Acad. Sci. USA 1973, 70, 1447-1449. 
26. Anderson, M.E. Glutathione: An overview of biosynthesis and modulation. Chem. Biol. Interact. 1998, 111-112, 1-14.

27. U.S. National Library of Medicine. Verteporfin Injection. Available online: http://www.nlm.nih. gov/medlineplus/druginfo/meds/a607060.html (accessed on 21 June 2015).

28. Novartis Company. Visudyne: Verteporfin. Available online: http://www.novartis.com.au/PI_PDF/ vid.pdf (accessed on 5 June 2015).

29. Pancewicz, S.A.; Skrzdlewska, E.; Hermanowska-Szpakowicz, T.; Zajkowska, J.M.; Kondrusik, M. Role of reactive oxygen species (ros) in patients with erythema migrans, an early manifestation of lyme borreliosis. Med. Sci. Monit. 2001, 7, 1230-1235.

30. Townsend, D.M.; Tew, K.D. The role of glutathione-s-transferase in anti-cancer drug resistance. Oncogene 2003, 22, 7369-7375.

31. Kensler, T.W.; Qian, G.S.; Chen, J.G.; Groopman, J.D. Translational strategies for cancer prevention in liver. Nat. Rev. Cancer 2003, 3, 321-329.

32. Siles, S.A.; Srinivasan, A.; Pierce, C.G.; Lopez-Ribot, J.L.; Ramasubramanian, A.K. High-throughput screening of a collection of known pharmacologically active small compounds for identification of Candida albicans biofilm inhibitors. Antimicrob. Agents Chemother. 2013, 57, 3681-3687.

33. Hayek, S.R.; Lee, S.A.; Parra, K.J. Advances in targeting the vacuolar proton-translocating ATPase (V-ATPase) for anti-fungal therapy. Front. Pharmacol. 2014, 5, doi:10.3389/fphar.2014.00004.

34. Chan, C.Y.; Prudom, C.; Raines, S.M.; Charkhzarrin, S.; Melman, S.D.; de Haro, L.P.; Allen, C.; Lee, S.A.; Sklar, L.A.; Parra, K.J. Inhibitors of V-ATPase proton transport reveal uncoupling functions of tether linking cytosolic and membrane domain of v0 subunit a (vph1p). J. Biol. Chem. 2012, 287, 10236-10250.

35. Fraser, C.M.; Casjens, S.; Hang, W.M.; Sutton, G.G.; Clayton, R.; Lathigra, R.; White, O.; Ketchum, K.A.; Dodson, R.; Hickey, E.K.; et al. Genomic sequence of a lyme disease spirochaete, Borrelia burgdorferi. Nature 1997, 390, 580-586.

36. Daull, P.; Lallemand, F.; Garrique, J.S. Benefits of cetalkonium chloride cationic oil-in-water nanoemulsions for topical opthalmic drug delivery. J. Pharm. Pharmacol. 2014, 66, 531-541.

37. Noecker, R. Effects of common ophthalmic preservatives on ocular health. Adv. Ther. 2001, 18, 205-215.

38. Cabo, M.L.; Herrera, J.J.; Crespo, M.D.; Pastoriza, L. Comparison among the effectiveness of ozone, nisin and benzalkonium chloride for the elimination of planktonic cells and biofilms of Staphylococcus aureus cect4459 on polypropylene. Food Control 2009, 20, 521-525.

39. Zhang, Y.; Wade, M.M.; Scorpio, A.; Zhang, H.; Sun, Z. Mode of action of pyrazinamide: Disruption of Mycobacterium tuberculosis membrane transport and energetics by pyrazinoic acid. J. Antimicrob. Chemother. 2003, 52, 790-795.

40. Niu, H.; Cui, P.; Shi, W.; Zhang, S.; Feng, J.; Wang, Y.; Sullivan, D.; Zhu, B.; Zhang, Y. Identification of anti-persister activity against uropathogenic Escherichia coli from a clinical drug library. Antibiotics 2015, 4, 179-187.

41. Hurdle, J.G.; O’Neill, A.J.; Chopra, I.; Lee, R.E. Targeting bacterial membrane function: An underexploited mechanism for treating persistent infections. Nat. Rev. Microbiol. 2011, 9, 62-75.

42. Feng, J., Shi, W., Zhang, S., Zhang, Y. Persister mechanisms in Borrelia burgdorferi: Implications for improved intervention. Emerg. Microbes Infect. 2015, 4, doi:10.1038/emi.2015.51. 
43. Sharma, B.; Brown, A.V.; Matluck, N.E.; Hu, L.T.; Lewis, K. Borrelia burgdorferi, the causative agent of lyme disease, forms drug-tolerant persister cells. Antimicrob. Agents Chemother. 2015, 59, 4616-4624.

44. Feng, J.; Wang, T.; Zhang, S.; Shi, W.; Zhang, Y. An optimized SYBR Green I/PI assay for rapid viability assessment and antibiotic susceptibility testing for Borrelia burgdorferi. PLoS ONE 2014, 9, e111809.

45. Baell, J.; Walters, M.A. Chemistry: Chemical con artists foil drug discovery. Nature 2014, 513, 481-483.

46. PUBCHEM PROMISCUITY. Available online: http://chemutils.florida.scripps.edu:8080/pcpro miscuity/pcpromiscuity.html (accessed on 5 June 2015).

(C) 2015 by the authors; licensee MDPI, Basel, Switzerland. This article is an open access article distributed under the terms and conditions of the Creative Commons Attribution license (http://creativecommons.org/licenses/by/4.0/). 